\title{
POLARIZATION OBSERVABLES IN THE ELASTIC SCATTERING OF PROTONS ON ${ }^{9}$ Be NUCLEI
}

\author{
Yu. A. Berezhnoy ${ }^{1}$, V. P. Mikhailyuk ${ }^{2}$ \\ ${ }^{1}$ Kharkiv National University, Kharkiv, UA-61077, Ukraine \\ ${ }^{2}$ Institute for Nuclear Research, Kyiv, UA-03680, Ukraine
}

(Received February 11, 2003; received in final form November 20, 2003)

\begin{abstract}
The polarization observables for the elastic $p^{9} \mathrm{Be}$ scattering at $1000 \mathrm{MeV}$ are calculated on the basis of the Glauber-Sitenko multiple diffraction scattering theory and $\alpha$-cluster model with dispersion. The ${ }^{9} \mathrm{Be}$ nucleus is considered as composed of the core $\left({ }^{8} \mathrm{Be}\right.$ nucleus $)$ and the additional cluster (neutron) arranged with most probability in the center of mass of the core. The results obtained are in agreement with the existing experimental data.

Key words: elastic scattering, polarization observables, high energies, $\alpha$-cluster model with dispersion, multiple diffraction scattering theory.
\end{abstract}

PACS number(s): 24.10.Ht, 25.45.De

\section{INTRODUCTION}

The investigation of the polarization phenomena in the elastic scattering of intermediate energy particles on light nuclei is an important test for studying scattering dynamics, nuclear structure and nucleon-nucleon (NN) interaction. Polarization observables can give more information about the structure of target nuclei and the mechanisms of nuclear reactions as compared with total, reaction, differential, cross-sections. For example, analyzing power (polarization) can be used to determine the imaginary part of spin-orbital nucleon-nucleus amplitude [1], and other observables are very sensitive to the choice of "elementary" amplitudes and target model.

In recent years the polarization phenomena in the elastic scattering of intermediate energy protons on even nuclei $\left({ }^{12} \mathrm{C},{ }^{16} \mathrm{O},{ }^{20} \mathrm{Ne}\right)$ are intensively investigated $[2-5]$. To describe the polarization observables in these processes the $\alpha$-cluster model with dispersion [2] has been proposed. According to this model the carbon and oxygen nuclei are considered as made up of three and four $\alpha$-clusters arranged at the vertices of equilateral triangle and tetrahedron, respectively. These $\alpha$-clusters can be displaced from their most probable equilibrium positions.

In $[4,5]$ the ${ }^{20} \mathrm{Ne}$ nucleus was considered as composed of the deformed core $\left({ }^{16} \mathrm{O}\right.$ nucleus $)$ and the additional $\alpha$-cluster situated with the most probability inside [4] and outside of the core [5]. The observables calculated by means of the model in which we suppose that the additional $\alpha$-cluster is situated with the most probability inside the core are in a better agreement with the experimental data as compared with those calculated in the model in which additional $\alpha$-cluster is situated outside the core.

By means of the $\alpha$-cluster model with dispersion and Glauber-Sitenko multiple diffraction scattering theory (MDST) [6,7] we have described the observables in the elastic $p{ }^{12} \mathrm{C}, p-^{16} \mathrm{O}$ and $p{ }^{20} \mathrm{Ne}$ scattering at intermedi- ate energies. The results of the calculations were in agreement with the experimental data. Moreover, we have shown that spin-rotation functions for the elastic scattering of protons on ${ }^{12} \mathrm{C}$ and ${ }^{16} \mathrm{O}$ nuclei differ qualitatively as compared with those calculated in the free-nucleon model [3].

Notice that for full description of the elastic scattering of $\frac{1}{2}$-spin protons on zero-spin nuclei it is necessary to measure three independent observables [1]. In the case of proton scattering on odd nuclei $\left({ }^{9} \mathrm{Be},{ }^{13} \mathrm{C},{ }^{13} \mathrm{~N}\right.$, etc.) the polarization phenomena are more various and intricate. So, for example, to describe the elastic scattering of two $\frac{1}{2}$-spin particles it is necessary to measure eleven independent observables [8].

Therefore, it would be interesting to investigate the scattering processes of protons on odd light nuclei. One of the most investigated odd nucleus is a ${ }^{9} \mathrm{Be}$ nucleus. In this paper we suppose the $2 \alpha n$ configuration for ${ }^{9} \mathrm{Be}$ nucleus. In other words we consider ${ }^{9} \mathrm{Be}$ nucleus as madeup of the core $\left({ }^{8} \mathrm{Be}\right.$ nucleus $)$ and the additional neutron situated with the most probability inside the core.

Notice that the three-particle wave functions of the ${ }^{9} \mathrm{Be}$ nucleus in the $2 \alpha n$ model with different pair potentials were calculated, for example, in [9]. Number of approaches to investigate the elastic scattering of protons by ${ }^{9} \mathrm{Be}$ nucleus at intermediate energies were used by many authors (see, for example, [10-15] and references therein). To describe the observables in the elastic $p-{ }^{9} \mathrm{Be}$ scattering the optical model [11-13], macroscopic DWIA $[10,12,13]$ and the coupled channel approximation [12] were used. The $2 \alpha n$ model and MDST were used in [14, $15]$ to describe the polarization observables in the elastic $p-{ }^{9} \mathrm{Be}$ scattering at 220 and $1000 \mathrm{MeV}$. The results obtained in $[14,15]$ are in agreement with experimental data.

In this paper the model analogous to proposed in [4] is developed for the case of ${ }^{9} \mathrm{Be}$ nuclei. In Section II the brief theoretical formalism is described, and in Section III the results obtained are discussed. 


\section{THEORETICAL FORMALISM}

The ${ }^{9}$ Be nucleus can be considered as made-up of the core $\left({ }^{8} \mathrm{Be}\right.$ nucleus $)$ and the additional neutron situated with the most probability inside the core. In this case we can choose the manyparticle density of ${ }^{9} \mathrm{Be}$ nucleus in the form

$$
\rho^{(\mathrm{Be})}(\boldsymbol{\xi}, \boldsymbol{\eta})=\rho_{\Delta}(\boldsymbol{\xi}) \rho_{(N)}(\boldsymbol{\eta})
$$

where $\rho_{\Delta}(\boldsymbol{\xi})$ is the density of the core $\left({ }^{8} \mathrm{Be}\right.$ nucleus); $\rho_{(N)}(\boldsymbol{\eta})$ is the density of the additional cluster (neutron); $\boldsymbol{\xi}$ is the ${ }^{9} \mathrm{Be}$ nucleus Jacobi coordinate of the $\alpha$-clusters; $\boldsymbol{\eta}$ is the coordinate of the additional neutron.

The density of the core can be presented in the form

$$
\begin{aligned}
& \rho_{\Delta}(\boldsymbol{\xi})=\int d^{3} \xi^{\prime} \rho_{0}\left(\boldsymbol{\xi}^{\prime}\right) \Phi_{\Delta}\left(\boldsymbol{\xi}-\boldsymbol{\xi}^{\prime}\right), \\
& \rho_{0}(\boldsymbol{\xi})=\frac{1}{4 \pi d^{2}} \delta(\xi-d), \\
& \Phi_{\Delta}(\xi)=\frac{1}{\left(2 \pi \Delta^{2}\right)^{\frac{3}{2}}} \exp \left(-\frac{\xi^{2}}{2 \Delta^{2}}\right) .
\end{aligned}
$$

The density $\rho_{0}(\boldsymbol{\xi})$ is normalized to unity and correspond to rigid dumb-bell composed of two $\alpha$-particles $\left({ }^{8}\right.$ Be nucleus). Smearing function $\Phi_{\Delta}(\boldsymbol{\xi})$ is normalized to unity and if $\Delta \rightarrow 0$

$$
\lim _{\Delta \rightarrow 0} \Phi_{\Delta}\left(\boldsymbol{\xi}-\boldsymbol{\xi}^{\prime}\right)=\delta\left(\boldsymbol{\xi}-\boldsymbol{\xi}^{\prime}\right) .
$$

In (2)-(4) parameters $\mathrm{d}$ and $\Delta$ characterize the distance between the $\alpha$-clusters and the probability of the $\alpha$-clusters displacement from its most probable position at the vertex of the dumb-bell, respectively.

Integrating (2) we have

$$
\rho_{\Delta}(\boldsymbol{\xi})=\frac{1}{\left(2 \pi \Delta^{2}\right)^{\frac{3}{2}}} \exp \left(-\frac{\xi^{2}}{2 \Delta^{2}}-\frac{d^{2}}{2 \Delta^{2}}\right) \frac{\sinh (x)}{x}
$$

where $\sinh (x)$ is the hyperbolical sine, $x=\frac{\xi d}{\Delta^{2}}$.

The density of the additional neutron we choose in the form

$$
\rho_{(N)}(\boldsymbol{\eta})=\frac{1}{(\lambda \sqrt{\pi})^{3}} \exp \left(-\frac{\eta^{2}}{\lambda^{2}}\right)
$$

where parameter $\lambda$ characterizes the distance between the additional neutron and the center of mass of the core.

The density parameters $d$ and $\Delta$ can be determined from the comparison of the calculated and measured elastic scattering charge formfactors of ${ }^{9} \mathrm{Be}$ nucleus. Taking into account that charge formfactor of neutron is equal to zero we can present the elastic scattering formfactor of ${ }^{9} \mathrm{Be}$ nucleus in the form

$$
F^{(\mathrm{Be})}(q)=\exp \left(-\frac{1}{6} q^{2}\left\langle r^{2}\right\rangle_{\alpha}-\frac{1}{8} q^{2} \Delta^{2}\right) j_{0}\left(\frac{q d}{2}\right)
$$

where $j_{0}(x)$ is the Bessel spherical function, $\left\langle r^{2}\right\rangle_{\alpha}^{1 / 2}=$ $1.61 \mathrm{Fm}$ is the root mean square radius of the $\alpha$-cluster, $\mathbf{q}$ is the transferred momentum.

Fig. 1 shows the calculated ${ }^{9} \mathrm{Be}$ elastic scattering charge form factor with the experimental data taken from $[16,17]$. As can be seen from Fig. 1 the calculated and measured form factors are in agreement up to the values of transferred momentum $q \leq 2 \mathrm{Fm}^{-1}$.

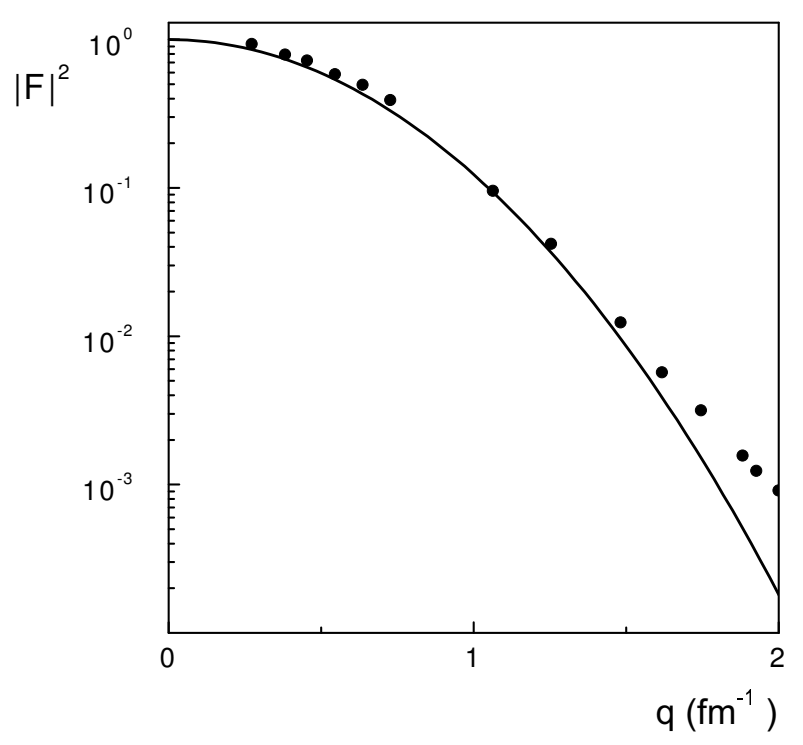

Fig. 1. The ${ }^{9}$ Be charge form factor as a function of the momentum transferred. The experimental data are from [16,17].

From the comparison of the calculated and measured form factors we have obtained the following values of the ${ }^{9}$ Be density parameters: $d=2.081 \mathrm{Fm}, \Delta=1.850 \mathrm{Fm}$.

In this approach the root mean square radius of ${ }^{9} \mathrm{Be}$ nucleus is determined by

$$
\left\langle r^{2}\right\rangle_{\mathrm{Be}}=\left\langle r^{2}\right\rangle_{\alpha}+\frac{1}{4} d^{2}+\frac{3}{4} \Delta^{2}
$$

The obtained values of density parameters for ${ }^{9} \mathrm{Be}$ nucleus yield the root mean square radius $\left\langle r^{2}\right\rangle_{\text {theor }}^{1 / 2}$ $=2.498 \mathrm{Fm}$ which is close to the experimentally measured value [18] $\left\langle r^{2}\right\rangle_{\exp }^{1 / 2}=2.519 \mathrm{Fm}$.

Notice that in the above approach the value of the parameter $\lambda$ can not be determined from the comparison of the measured and calculated charge form factors of ${ }^{9} \mathrm{Be}$ nuclei. To calculate the observables in the elastic scattering of protons on ${ }^{9} \mathrm{Be}$ nucleus we used the value of the parameter $\lambda=1.23 \mathrm{Fm}$, which is aproximately average value of the same parameter obtained in $[4,5]$ for ${ }^{20} \mathrm{Ne}$ nuclei. 
As it can be seen from the above approach the proposed for ${ }^{9} \mathrm{Be}$ nucleus model is sufficiently simple. In this $\alpha$-cluster model with dispersion we have neglected of the exchange of nucleons between $\alpha$-clusters during the interactions of incident protons with ${ }^{9} \mathrm{Be}$ nucleus. However, the results obtained in $[2,3]$ show that at the region of considered energy due to the smallness of interaction time of incident particle with nucleus as compared with the internal nucleon motion, the exchange of nucleons between $\alpha$-clusters can be neglected. In other words, incident proton have no time to "see" the exchange of nucleons between $\alpha$-clusters because this processes takes place sufficently seldom and slowly. Moreover, the energy of the incident particle is much higher than the Fermi energy, so at the elastic scattering of intermediate energy particle by light nucleus the correlation effects between the target nucleons due to the Pauli principle cannot take significant role.

Consider elastic scattering of protons on ${ }^{9} \mathrm{Be}$ nucleus. According to MDST the $p-{ }^{9} \mathrm{Be}$ elastic scaterring amplitude is determined through the elementary proton$\alpha$ and proton-neutron amplitudes. In the most general form nucleon-nucleon amplitude $f_{\mathrm{N}}(q)$ has the form

$$
\begin{aligned}
& f_{\mathrm{NN}}(q)=f_{1}(\mathbf{q})+q f_{2}(\mathbf{q})\left(\boldsymbol{\sigma}_{0} \mathbf{n}+\boldsymbol{\sigma}_{1} \mathbf{n}\right)+f_{3}(\mathbf{q})\left(\boldsymbol{\sigma}_{0} \boldsymbol{\sigma}_{1}\right) \\
& +f_{4}(\mathbf{q})\left(\boldsymbol{\sigma}_{0} \mathbf{q}\right)\left(\boldsymbol{\sigma}_{1} \mathbf{q}\right)+f_{5}(\mathbf{q})\left(\boldsymbol{\sigma}_{0} \mathbf{p}\right)\left(\boldsymbol{\sigma}_{1} \mathbf{p}\right)
\end{aligned}
$$

where $\sigma_{0}$ and $\sigma_{1}$ are the spin operators of the incident proton and additional neutron of the target nucleus, $\mathbf{n}=\left[\mathbf{k}, \mathbf{k}^{\prime}\right] /\left|\left[\mathbf{k}, \mathbf{k}^{\prime}\right]\right|, \mathbf{q}=\mathbf{k}-\mathbf{k}^{\prime}, \mathbf{p}=\left(\mathbf{k}+\mathbf{k}^{\prime}\right) /\left(\left|\mathbf{k}+\mathbf{k}^{\prime}\right|\right)$, $\mathbf{k}$ and $\mathbf{k}^{\prime}$ are the wave-vectors of the incident and scattered protons. The vectors $\mathbf{n}, \mathbf{p}$ and $-\mathbf{q} /|\mathbf{q}|$ form the right-hand orthogonal coordinate system. Neglecting the isospin part of the $\mathrm{NN}$ interaction we choose the amplitudes $f_{i}(\mathbf{q})$ in the form

$$
f_{i}(\mathbf{q})=k H_{i} \exp \left(-\gamma_{i} q^{2}\right), \quad 1 \leq i \leq 5
$$

The numerical values of parameters $H_{i}, \gamma_{i}$ obtained from the phase-shift solutions are presented in [8].

Elementary proton $-\alpha$ amplitude can be chosen in the form [3]

$$
\begin{aligned}
f_{p \alpha}(\mathbf{q}) & =k \sum_{i=1}^{2}\left(G_{c i} \exp \left(-\beta_{c i} q^{2}\right)\right. \\
& \left.+q G_{s i} \exp \left(-\beta_{s i} q^{2}\right)\left(\boldsymbol{\sigma}_{0} \mathbf{n}\right)\right)
\end{aligned}
$$

The parameters $G_{c 1}, \beta_{c 1}, G_{s 1}$ and $\beta_{s 1}$ are the fitting ones, and parameters $G_{c 2}, \beta_{c 2}, G_{s 2}$ and $\beta_{s 2}$ are related with $G_{c 1}, \beta_{c 1}, G_{s 1}$ and $\beta_{s 1}$ through [3]

$$
\begin{aligned}
G_{c 2} & =\frac{3 i G_{c 1}^{2}}{32 \beta_{c 1}}, \quad \beta_{c 2}=\frac{1}{2} \beta_{c 1}, \\
G_{s 2} & =\frac{3 i G_{c 1} G_{s 1} \beta_{c 1}}{8\left(\beta_{c 1}+\beta_{s 1}\right)^{2}}, \quad \beta_{s 2}=\frac{\beta_{c 1} \beta_{s 1}}{\beta_{c 1}+\beta_{s 1}} .
\end{aligned}
$$

The values of the parameters $G_{c 1}, \beta_{c 1}, G_{s 1}$ and $\beta_{s 1}$ obtained from the comparison of the calculated and measured [19] elastic scattering $p-{ }^{4} \mathrm{He}$ observables at $1000 \mathrm{MeV}$ are $: G_{c 1}=0.336+i 1.277\left(\mathrm{Fm}^{2}\right), \beta_{c 1}=$ $0.435-i 0.029\left(\mathrm{Fm}^{2}\right), G_{s 1}=0.179+i 0.215\left(\mathrm{fm}^{3}\right)$ and $\beta_{s 1}=0.476+i 0.013\left(\mathrm{Fm}^{2}\right)$.

Elastic scattering $p-{ }^{9} \mathrm{Be}$ amplitude can be presented in the form

$$
\begin{aligned}
F^{(\mathrm{Be})}(\mathbf{q}) & =A+E\left(\boldsymbol{\sigma}_{0} \mathbf{n}\right)+F\left(\boldsymbol{\sigma}_{1} \mathbf{n}\right)+B\left(\boldsymbol{\sigma}_{0} \mathbf{n}\right)\left(\boldsymbol{\sigma}_{1} \mathbf{n}\right) \\
& +C\left(\boldsymbol{\sigma}_{0} \mathbf{q}\right)\left(\boldsymbol{\sigma}_{1} \mathbf{q}\right)+D\left(\boldsymbol{\sigma}_{0} \mathbf{p}\right)\left(\boldsymbol{\sigma}_{1} \mathbf{p}\right)
\end{aligned}
$$

Polarization observables for the elastic $p{ }^{9}$ Be scattering calculated in the above approach are determined through the amplitudes $A, E, F, B, C$ and $D$.

\section{DISCUSSION}

As was mentioned above for full description of the elastic scattering of two $\frac{1}{2}$-spin particles it is necessary to measure eleven independent observables [8]. The ${ }^{9} \mathrm{Be}$ nucleus in ground state has spin $I=\frac{3}{2}^{-}$. Therefore number of independent observables which form the complete set must increases.

At present a more complete experimental data for the elastic scattering of protons on odd nuclei exist for $p$ ${ }^{13} \mathrm{C}$ scattering at $500 \mathrm{MeV}[20-22]$. In these papers the differential cross section $\sigma(\theta) \equiv \frac{d \sigma}{d \Omega}(\mathrm{mb} / \mathrm{sr})$, polarization (asymmetry) $P(\theta)$, spin-rotation-depolarization parameters $D_{\mathrm{LS}}(\theta), D_{\mathrm{SS}}(\theta), D_{\mathrm{NN}}(\theta)$, target analyzing power $A_{000 \mathrm{~N}}(\theta)$ and spin correlation $A_{00 \mathrm{NN}}(\theta)$ have been measured. These observables are determined by [23]

$$
\begin{aligned}
& \sigma=|A|^{2}+|B|^{2}+|C|^{2}+|D|^{2}+|E|^{2}+|M|^{2}, \\
& \sigma P=2 \operatorname{Re}\left(A^{*} E+B^{*} M\right), \\
& \sigma D_{\mathrm{LS}}=2 \operatorname{Re}\left(A^{*} E-B^{*} M\right), \\
& \sigma D_{\mathrm{SS}}=|A|^{2}-|B|^{2}+|C|^{2}-|D|^{2}-|E|^{2}+|M|^{2}, \\
& \sigma D_{\mathrm{NN}}=|A|^{2}+|B|^{2}-|C|^{2}-|D|^{2}+|E|^{2}+|M|^{2}, \\
& \sigma A_{000 \mathrm{~N}}=2 \operatorname{Re}\left(A^{*} M+B^{*} E\right), \\
& \sigma A_{00 \mathrm{NN}}=2 \operatorname{Re}\left({ }^{*} B-C^{*} D+E^{*} M\right) .
\end{aligned}
$$

In present paper we calculated the same observables for $p-{ }^{9} \mathrm{Be}$ elastic scattering. In the calculations we used the parameters of the $p-\alpha$ amplitude determined in present paper and the parameters of proton-neutron amplitude determined in [8] at $800 \mathrm{MeV}$. The results obtained are presented in Figs. 2, 3 (dashed curves). As can be seen from Fig. 2 the calculated differential crosssection are in agreement with experimental data [24,25], and analyzing power (polarization) are in quantitative agreement with the existing data. 

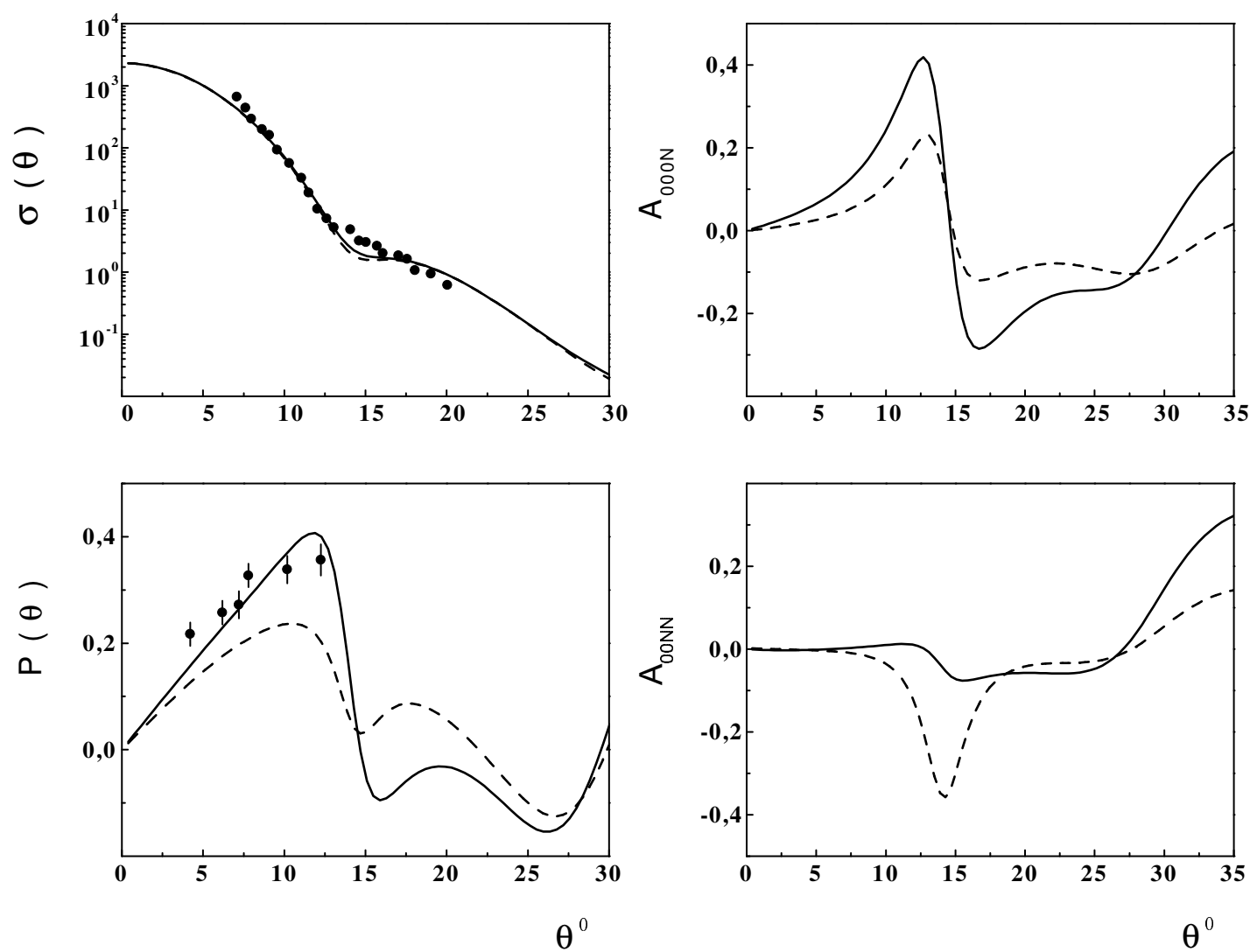

Fig. 2. Differential cross-section $\sigma(\theta)$, polarization $P(\theta)$, target analyzing power $A_{000 \mathrm{~N}}(\theta)$ and spin correlation $A_{00 \mathrm{NN}}(\theta)$ of the elastic $p-{ }^{9} \mathrm{Be}$ scattering at $1000 \mathrm{MeV}$ as a function of the scattering angle. Experimental data are from [24,25].

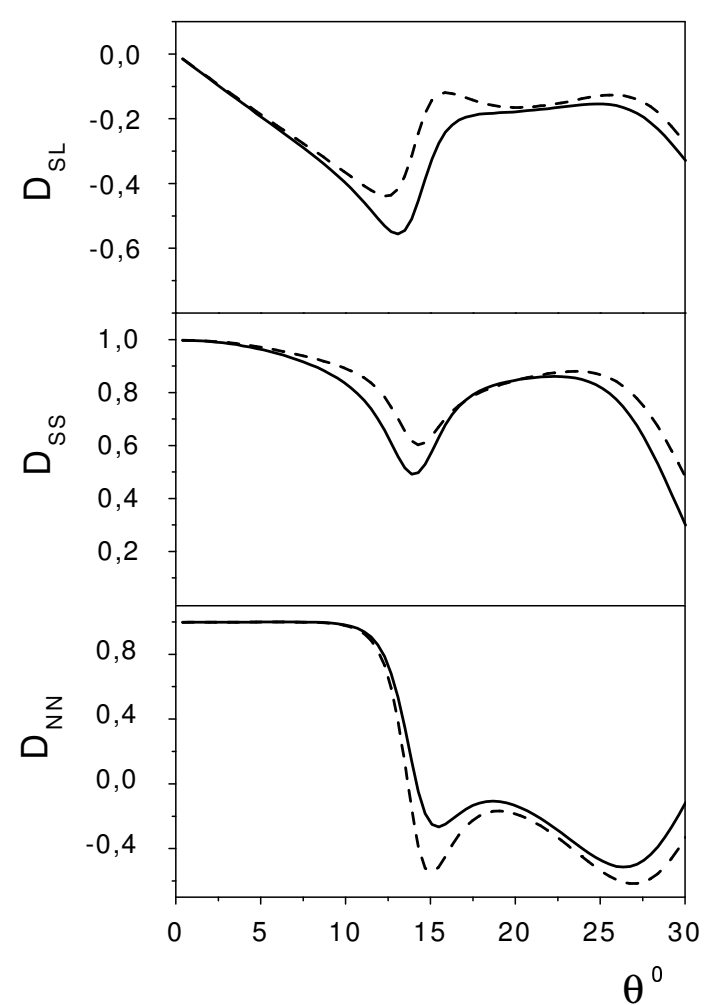

Fig. 3. Spin-rotation-depolarization parameters $D_{L S}(\theta)$, $D_{S S}(\theta)$ and $D_{N N}(\theta)$ of the elastic $p-{ }^{9} \mathrm{Be}$ scattering at $1000 \mathrm{MeV}$ as a function of the scattering angle.
As was mentioned above analyzing power (polarization) can be used to determine the imaginary part of spin-orbital nucleon-nucleus amplitude [1]. To describe the existing data (Figs. 2, 3 solid curves) we change the imaginary part of spin-orbital $\mathrm{NN}$ amplitude $\left(\mathrm{Im} \mathrm{H}_{2}=\right.$ $-4.51(\mathrm{GeV} / \mathrm{c})^{-3}[8]$ and $\operatorname{Im} H_{2}=-10.51(\mathrm{GeV} / \mathrm{c})^{-3}$ in present paper).

As can be seen from Figs. 2, 3 the behaviour of the calculated observables for the elastic $p{ }^{9}$ Be scattering is similar to those for $p{ }^{13} \mathrm{C}$ scattering [20-22] right up to $\theta \sim 15^{\circ}$. Then the calculated in the present paper and measured in [20-22] observables differ quantitatively. It can be due to the fact that ${ }^{9} \mathrm{Be}$ nucleus is weakly bounded than ${ }^{13} \mathrm{C}$ nucleus and with the increasing of the momentum transferred increases influence of the nucleon degree of freedom on the behaviour of the observables. Moreover, the model proposed have some imperfections. So, in the model $[4,5]$ the removing of the additional $\alpha$ cluster from ${ }^{20} \mathrm{Ne}$ nucleus to infinity leads to the transformation of ${ }^{20} \mathrm{Ne}$ nucleus to the stable ${ }^{16} \mathrm{O}$ nucleus. In contrast, the removing of the additional neutron from ${ }^{9}$ Be nucleus leads to the transformation of this nucleus to the unstable ${ }^{8}$ Be nucleus.

Notice that the $2 \alpha n$ model and MDST was used in $[14,15]$ to describe the polarization observables in the elastic $p{ }^{9}$ Be scattering at 220 and $1000 \mathrm{MeV}$. In [14,15] the ground state ${ }^{9} \mathrm{Be}$ wave function was presented as an 
expansion of Gaussians, and the NN amplitude was taken as a sum of central and spin-orbital interaction. The results obtained in $[14,15]$ are in agreement with experimental data. Unfortunately, in $[14,15]$ only differential cross-section and analyzing power up to $\theta \sim 20^{\circ}$ were calculated. Therefore, it is impossible to properly compare the results obtained in present paper and those in $[14,15]$

Therefore, we conclude that assumption about the model in which ${ }^{9} \mathrm{Be}$ nucleus is considered as made-up of the core ( ${ }^{8} \mathrm{Be}$ nucleus) and the additional neutron situated with the most probability inside the core allows us to agree the calculated and measured charge formfactors up to the values of transferred momentum $q \leq 2 \mathrm{Fm}^{-1}$, root mean square radius of ${ }^{9} \mathrm{Be}$ nucleus and observables in the elastic $1-\mathrm{GeV}$ proton elastic scattering on these nuclei. The results obtained in this paper and by other authors (see, for example [9-15] and references therein) show that $2 \alpha n$ configuration is presented with great probability in the wave function of ${ }^{9} \mathrm{Be}$ nucleus.

The experimental measurements of the maximally possible number of independent observables in maximally possible angle range could serve as a more critical verification of the models which describe nucleon-nucleus interactions and it provide more information about nuclear structure and nature of nuclear forces.
[1] P. Osland, R. G. Glauber, Nucl. Phys. A 326, 225 (1979).

[2] Yu. A. Berezhnoy, V. V. Pilipenko, G. A. Khomenko, J. Phys. G 10, 63 (1984).

[3] Yu. A. Berezhnoy, V. P. Mikhailyuk, V. V. Pilipenko, J. Phys. G 18, 85 (1992).

[4] Yu. A. Berezhnoy, V. P. Mikhailyuk, Int. J. Mod. Phys. E 8, 1 (1999).

[5] Yu. A. Berezhnoy, V. P. Mikhailyuk, Izv. RAN, Ser. fiz. 65, 721 (2001).

[6] R. J. Glauber, Lect. in Theor. Phys. V. 1, edited by W. E. Brittin, L. G. Dunham, (New-York, Intersci, 1959), p. 315.

[7] O. G. Sitenko, Ukr. Phys. J. 4, 152 (1959).

[8] I. N. Kudryavtsev, A. P. Soznik, J. Phys. G 15, 1377 (1989).

[9] V. T. Voronchev, V. I. Kukulin, Few-body Systems 18, 191 (1995).

[10] S. Dixit et al., Phys. Rev. C 43 , 1758 (1991).

[11] C. W. Glover et. al., Phys. Rev. C 43 , 1664 (1991).
[12] G. Roy, Nucl. Phys. A 442, 686 (1985).

[13] D. K. Hassel, Phys. Rev. C 34, 236 (1986).

[14] M. A. Zhusupov, E. T. Ibraeva, Sov. J. Nucl. Phys. 61, 51 (1998).

[15] M. A. Zhusupov, E. T. Ibraeva, Phys. Elementary Part. Atom. Nucl. 31, 1427 (2000)

[16] G. D. Alkhazov, O. A. Domchenkov, Sov. J. Nucl. Phys. 37, 84 (1983).

[17] J. P. Glickman, Phys. Rev. C 43, 1740 (1991).

[18] H. de Vries, C. W. de Jager, C. de Vries, At. Data Nucl. Data Tables 36, 495 (1987).

[19] H. Courant, Phys. Rev. C 19, 104 (1979).

[20] G. W. Hoffman, Phys. Rev. C 41, 1651 (1990).

[21] G. W. Hoffman, Phys. Rev. Lett. 65, 3096 (1990).

[22] G. W. Hoffman, Phys. Rev. C 53, 1974 (1996).

[23] L. Ray, Phys. Rev. C 37, 1169 (1988).

[24] G. D. Alkhazov, Sov. J. Nucl. Phys. 42, 8 (1985).

[25] V. G. Vovchenko, Sov. J. Nucl. Phys. 16, 1145 (1972).

\title{
ПОЛЯРИЗАЦІЙНІ ХАРАКТЕРИСТИКИ ПРУЖНОГО РОЗСІЯННЯ ПРОТОНІВ ЯДРАМИ ${ }^{9} \mathrm{Be}$
}

\author{
Ю. А. Бережной ${ }^{1}$, В. П. Михайлюк ${ }^{2}$ \\ ${ }^{1}$ Харківсъкий начіоналъний університет, пл. Свободи, 4, Харків, 61077, Украӥна \\ ${ }^{2}$ Iнститут ядерних досліджень НАН України, просп. Науки, 47, Киӥв, 03680, Украӥна
}

\begin{abstract}
Поляризаційні характеристики пружного $p^{-9}$ Ве розсіяння при енергіï $1000 \mathrm{MeB}$ розраховано на основі теорії багатократного дифракційного розсіяння Глаубера-Ситенка й $\alpha$-кластерної моделі з дисперсією. Ядро ${ }^{9}$ Ве розглянуто таким, що складається з кору (ядро ${ }^{8} \mathrm{Be}$ ) і додаткового кластера (нейтрона), який 3 найбільшою ймовірністю знаходиться в центрі мас кору. Отримані результати дають змогу описати наявні експериментальні дані.
\end{abstract}

